\title{
Ensamble de estructuras a nivel mesoscópico: formación de trímeros no-simétricos
}

\section{Structures assembly in the mesoscale: non-symmetric trimers formation}

\author{
Andrés Arango-Restrepo ${ }^{1}$, Daniel Barragán ${ }^{2}$ \\ ${ }^{1}$ Grupo de investigación en Calorimetría y Termodinámica de Procesos Irreversibles, Universidad Nacional de Colombia, \\ Medellín, Colombia. Orcid: 0000-0002-0076-5244.e-mail: aarangor@unal.edu.co \\ ${ }^{2}$ Escuela de Química, Facultad de Ciencias, Universidad Nacional de Colombia, Medellín, Colombia. \\ Orcid: 0000-0002-7390-1104. e-mail: dalbarraganr@unal.edu.co
}

RECIBIDO: Marzo 11, 2017. ACEPTADO: Junio 14, 2017. Versión FINAL: Octubre 30, 2017.

\begin{abstract}
RESUMEN
El ensamble de estructuras se observa desde la escala molecular hasta la cosmológica. El ensamble de componentes discretos fundamentales puede llevar a la formación de una amplia variedad de estructuras, desde simples hasta complejas. En este trabajo estudiamos el ensamble de bloques moleculares fundamentales que forman trímeros nosimétricos en la mesoescala. El proceso de ensamble se modela mediante un sistema de ecuaciones tipo Fokker-Planck acopladas en la frontera. El modelo matemático se resuelve en un espacio de estados, donde las trayectorias dan información de la probabilidad de formar diferentes trímeros por difusión a través de una barrera de potencial de energía no simétrica. Los resultados muestran que las condiciones de frontera no-homogéneas y la configuración de la barrera de potencial determinan cual estructura ensamblada, trímero, tiene la mayor probabilidad de formarse.
\end{abstract}

PALABRAS CLAVE: Ensamble de estructura; fokker-planck; mesoescala; termodinámica de no-equilibrio.

\begin{abstract}
Structures assembly takes place from molecular to cosmological scale. The building of structures comes up from fundamental and discrete components. In this work we study the formation of molecular non-symmetric trimers at the mesoscale. The assembly process is modeled by a set of Fokker-Planck type equations coupled through the boundary conditions. The mathematical model is solved in the space of trajectories where the probability of assembling is evaluated. Obtained results show how the assembly process depends on the boundary conditions and the shape of energy barriers.
\end{abstract}

KEYWORDS: Fokker-planck; mesoscale; non-equilibrium thermodynamics; structures assembly.

\section{INTRODUCCIÓN}

Los procesos de auto-ensamble se encuentran en la naturaleza y son importantes en aplicaciones tecnológicas [1]. Estos procesos se llevan a cabo fuera del equilibrio termodinámico [2], a partir de bloques fundamentales discretos [3] y tienen lugar en sistemas diferentes escalas: molecular elemental (moléculas),

ISSN Impreso: 1657 - 4583, En Línea: 2145 - 8456

Este artículo puede compartirse bajo la licencia CC BY-ND 4.0 y se referencia usando el siguiente formato: A. Arango, D. Barragán, "Ensamble de estructuras a nivel mesoscópico: formación de trímeros no-simétricos," Rev. UIS Ing., vol. 17, no. 1, pp. 163-168, 2018. Doi: https://doi.org/10.18273/revuin.v17n1-2018015 
molecular (cristales), planetaria (climática) [4], e incluso hasta cosmológica (anillos de Saturno) [5].

En los últimos años el interés en los procesos de autoensamble ha crecido en importancia, particularmente en la química supramolecular, la física de procesos tecnológicos, la biología celular [3] y ciencia de materiales [6]. Sin embargo, se conoce muy poco sobre los aspectos fundamentales que subyacen en estos procesos y no se cuenta con una teoría que permita su estudio a partir del ensamble de componentes discretos [2]. En este sentido es necesario contar con marco conceptual que permita comprender los aspectos dinámicos y termodinámicos que gobiernan la evolución del sistema durante el proceso de ensamblaje de estructuras a nivel mesoscópico.

Algunos trabajos previos en sistemas magnetohidrodinámicos desarrollaron modelos de ensamble basados en las ecuaciones clásicas de la hidrodinámica [7-8]. Sin embargo, lo usual es encontrar que para estudiar este tipo de procesos se recurra al modelado computacional mediante métodos Montecarlo y de Dinámica Molecular [9]. El principal objetivo de este trabajo es presentar un método con fundamentos teóricos, que permita estudiar y analizar el ensamble de estructuras tanto en sus aspectos dinámicos como termodinámicos. El método, que se fundamenta en la termodinámica mesoscópica de no-equilibrio [10], se constituye en una valiosa herramienta para modelar el ensamblaje de estructuras de diversa complejidad en sistemas fisicoquímicos fuera del equilibrio. En este trabajo se estudia el ensamblaje de trímeros moleculares que se obtienen por difusión a través de barreras de energía potencial no-simétricas; todo el proceso tiene lugar en la meso-escala.

\section{MÉTODOS}

La formación de trímeros moleculares a partir de componentes fundamentales discretos se modela mediante la termodinámica mesoscópica de no-equilibrio (MNET, siglas en inglés), obteniéndose un sistema de ecuaciones tipo Fokker-Planck (FP) [10]. Las barreras de potencial energético $\left(\phi_{i}\right)$ del proceso de ensamble se modelan como funciones bi-estables [11]. A partir de la solución del sistema de ecuaciones tipo FP se encuentra la densidad de probabilidad $p_{i}$ de encontrar en el sistema una determinada estructura: monómeros (bloque o componente fundamental), dímeros y trímeros.

Para el proceso de formar la estructura se considera que cada componente fundamental tiene dos formas de ensamblarse, así en el sistema se pueden encontrar durante el proceso siete posibles diferentes estructuras: 4 como trímeros, 2 como dímeros y componentes fundamentales. Durante el proceso los monómeros se ensamblan para dar dímeros y los dímeros se ensamblan con monomeros para dar trímeros. Según lo anterior, la probabilidad de encontrar las diferentes estructuras en el sistema está en función de dos variables internas independientes $\left(\gamma_{i, 1}, \gamma_{i, 2}\right)$ y una temporal $(t)$. Adicionalmente, el proceso de ensamble de las estructuras obedece una regla simple: una estructura no puede tener dos estados en un mismo proceso. Es decir, el ensamble sigue unas determinadas trayectorias de modo que si una estructura pertenece a $\gamma_{i, 1}>0$ entonces $\gamma_{i, 2}$ debe ser cero, o si la estructura pertenece a $\gamma_{i, 2}>0$ entonces $\gamma_{i, 1}$ debe ser cero.

El modelo propuesto consta de tres ecuaciones 2-D (una por cada proceso de ensamble), cada una de ellas en el espacio de fases de cada etapa del ensamble $\boldsymbol{\Gamma}_{i}=$ $\left(\gamma_{i, 1}, \gamma_{i, 2}\right)$ (para $\left.i=1,2,3\right)$, con condiciones de frontera acopladas tipo Neumann y con condición inicial diferente de cero únicamente para el componente fundamental.

\subsection{Ecuación tipo Fokker-Planck}

La ecuación tipo Fokker Planck para el $i$-ésimo proceso de ensamble viene dada por:

$$
\frac{\partial p_{i}\left(\boldsymbol{\Gamma}_{i}, t\right)}{\partial \mathrm{t}}=D\left[\nabla_{\boldsymbol{\Gamma}_{i}}^{2} p_{i}+\frac{1}{k_{B} T} \nabla_{\boldsymbol{\Gamma}_{i}}\left(p_{i} \nabla_{\boldsymbol{\Gamma}_{i}} \phi_{i}\left(\boldsymbol{\Gamma}_{i}\right)\right)\right],
$$

donde $D$ es la difusividad a través de las barreras de potencial energético $\phi_{i}$. Para el sistema en estudio se tienen 3 ecuaciones tipo Fokker-Planck (una por cada espacio) describiendo la formación de todas las posibles estructuras.

\subsection{Condición inicial}

La condición inicial del proceso de ensamble se define como:

$$
\begin{aligned}
& p_{i}\left(\gamma_{i, 1}, \gamma_{i, 2}, 0\right) \\
& =\left\{\begin{array}{cl}
0, & i \neq 1 \text { ó }\left(\gamma_{i, 1}, \gamma_{i, 2}\right) \neq(0,0) \\
\delta(0,0) & i=1 y\left(\gamma_{i, 1}, \gamma_{i, 2}\right)=(0,0)
\end{array}\right.
\end{aligned}
$$

\subsection{Condiciones de frontera}

Las condiciones de frontera son tipo Neumann homogéneas, excepto en los puntos en donde estas se acoplan, es decir los puntos para pasar del espacio $\boldsymbol{\Gamma}_{1}$ al espacio $\boldsymbol{\Gamma}_{i}$, para $i=2,3$. En otras palabras, estas condiciones de frontera reflejan el hecho de que existe un flujo de estados, desde $\boldsymbol{\Gamma}_{1}$, que se inducen debido al efecto de un bloque fundamental para estar disponibles a ensamblarse en los espacios $\boldsymbol{\Gamma}_{2}$ y $\boldsymbol{\Gamma}_{3}$. 
Al componente fundamental lo llamamos monómero $A$, representado por $p_{1}(0,0, t)$. El dímero $B$ está representado por $p_{1}(1,0, t)$, y el dímero $C$ por $p_{1}(0,1, t)$. Las condiciones de frontera no-homogéneas corresponden al flujo de dímero $B$ induciéndose para para ensamblarse en los trímeros $D$ y $E\left(J_{1}\right)$, y el flujo de dímero $C$ induciéndose para para ensamblarse en los trímeros $G$ y $F\left(J_{2}\right)$. Donde los trímeros $D, E, G$ y $F$ están representados por $p_{2}(1,0, t), p_{3}(0,1, t)$, y $p_{3}(1,0, t)$ respectivamente.

En la Figura 1 se ilustra la configuración de los espacios con los flujos entre las fronteras. Cada espacio es visto como un plano, en el cual solo tienen lugar los procesos en las trayectorias $T_{1, i}$ y $T_{2, i}$, donde la trayectoria 1 está dada por $T_{1, i} \mid \gamma_{i, 1}>0 \cap \gamma_{i, 2}=0$, y la trayectoria 2 está dada por $T_{2, i} \mid \gamma_{i, 2}>0 \cap \gamma_{i, 1}=0$.

A continuación se muestran las condiciones de frontera no-homogéneas. Como mencionamos, estas condiciones son generadas por el componente fundamental, el cual induce a los dímeros a salir del espacio $\boldsymbol{\Gamma}_{1}$ hacia los espacios $\begin{array}{lllll}\boldsymbol{\Gamma}_{2} & \text { y } & \boldsymbol{\Gamma}_{3} & \text { para realizar el ensamble de los }\end{array}$ trímeros:

Inducción del componente fundamental al dímero $B$ para salir del espacio $\boldsymbol{\Gamma}_{1}$ al $\boldsymbol{\Gamma}_{2}$ :

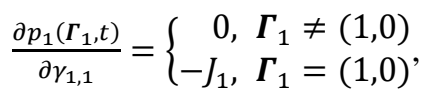

donde $J_{1}$ es el flujo de dímero $B$ entrando al espacio $\boldsymbol{\Gamma}_{2}$. A continuación se define la entrada del dímero $B$ al espacio $\boldsymbol{\Gamma}_{2}$ :

$$
\frac{\partial p_{2}\left(\boldsymbol{\Gamma}_{2}, t\right)}{\partial \gamma_{2,1}}= \begin{cases}0, & \boldsymbol{\Gamma}_{2} \neq(0,0) \\ J_{1}, & \boldsymbol{\Gamma}_{2}=(0,0)\end{cases}
$$

Inducción del componente fundamental al dímero $\mathrm{C}$ para salir del espacio $\boldsymbol{\Gamma}_{1}$ al $\boldsymbol{\Gamma}_{3}$ :

$$
\frac{\partial p_{1}\left(\boldsymbol{\Gamma}_{1}, t\right)}{\partial \gamma_{1,2}}=\left\{\begin{array}{rl}
0, & \boldsymbol{\Gamma}_{1} \neq(0,1) \\
-J_{2}, & \boldsymbol{\Gamma}_{1}=(0,1)
\end{array},\right.
$$

donde $J_{2}$ es el flujo de dímero $B$ entrando al espacio $\boldsymbol{\Gamma}_{3}$. A continuación se define la entrada del dímero $B$ al espacio $\boldsymbol{\Gamma}_{3}$ :

$$
\frac{\partial p_{3}\left(\boldsymbol{\Gamma}_{3}, t\right)}{\partial \gamma_{3,1}}= \begin{cases}0, & \boldsymbol{\Gamma}_{3} \neq(0,0) \\ J_{2}, & \boldsymbol{\Gamma}_{3}=(0,0)\end{cases}
$$

Los flujos $J_{1}$ y $J_{2}$ se definen como:

$$
\begin{aligned}
& J_{1}=D\left(p_{1}(0,0, t) p_{1}(1,0, t)-p_{2}(0,0, t)\right) \\
& J_{2}=D\left(p_{1}(0,0, t) p_{1}(0,1, t)-p_{3}(0,0, t)\right)
\end{aligned}
$$

Finalmente, para la solución numérica del sistema de ecuaciones se emplea el método de volúmenes finitos, donde se tiene especial cuidado al implementar las condiciones de frontera para así evidenciar el acople.

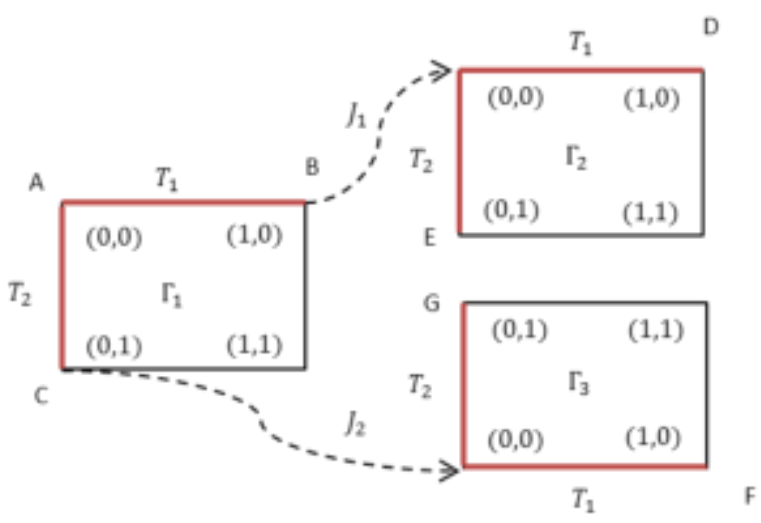

Figura 1. Ilustración de los espacios donde se lleva a cabo los diferentes pasos del ensamble. Fuente. Elaboración propia.

\subsection{Potenciales y probabilidades globales}

El modelamiento de los potenciales se realiza con polinomios de $5^{\text {to }}$ grado, tal que solo existan dos mínimos y un máximo en $\gamma_{i, j} \in\left[\begin{array}{ll}0 & 1\end{array}\right]$ [11]. Los valores de los potenciales químicos estándar y de las energías de activación $\left(\max \left(\phi_{i}\right)-\phi_{i}(0,0)\right)$ se presentan en la Tabla. 1. Los valores mostrados hacen referencia a procesos espontáneos, donde el primer proceso (ensamble del dímero) es el menos impedido energéticamente (menor energía de activación) y el tercer proceso (dímero $C$ a dímero $F$ y $G$ ) el más favorable termodinámicamente (mayor diferencia entre potenciales

\begin{tabular}{|c|c|c|c|}
\hline \multicolumn{4}{|c|}{ Valores termodinámicos claves de los potenciales } \\
\hline$i$ & 1 & 2 & 3 \\
\hline $\begin{array}{l}{\left[\max \left(\phi_{i}\left(\gamma_{i, 1}, 0\right)\right)-\phi_{i}(0,0)\right.} \\
\left.\max \left(\phi_{i}\left(\gamma_{i, 2}, 0\right)\right)-\phi_{i}(0,0)\right] / R T\end{array}$ & {$\left[\begin{array}{ll}1 & 1\end{array}\right]$} & {$\left[\begin{array}{ll}3 & 2\end{array}\right]$} & {$\left[\begin{array}{ll}2 & 2\end{array}\right]$} \\
\hline$\left[\phi_{i}\left(\gamma_{i, 1}, 0\right) \quad \phi_{i}\left(0, \gamma_{i, 2}\right)\right] / R T$ & $\begin{array}{c}- \\
{\left[\begin{array}{ll}1 & 2\end{array}\right]}\end{array}$ & $-\left[\begin{array}{ll}2 & 2\end{array}\right]$ & $-\left[\begin{array}{ll}3 & 4\end{array}\right]$ \\
\hline$\phi_{i}(0,0)$ & 0 & -1 & -2 \\
\hline
\end{tabular}
químicos estándar). La expresión trímeros no-simétricos hace referencia que el ensamble se lleva a cabo a través de barreras energéticas de potencial diferentes; todos los potenciales son energéticamente diferentes.

Tabla 1. Datos de los potenciales $\phi_{i}$.

Fuente. Elaboración propia. 
Notese que el estado del monómero $A$ corresponde a $p_{1}(0,0, t)$, un punto en particular. Sin embargo, en la mesoescala hay un continuo entre estados diferenciables, por lo que para $\gamma_{i, 1}$ y $\gamma_{i, 2}$ diferentes de 1 y 0 , no tenemos un estado en particular (monomero, dímero, o trímero) sino un estado intermedio. Las probabilidades globales recogen todos esos estados intermedios y los aproximamos al estado energéticamente más parecido [12]. Las probabilidades globales se definen como:

$$
\begin{aligned}
P_{A}(\mathrm{t})= & \sum_{j=1}^{2} \int_{0}^{\gamma_{1, j}^{*}} p_{1}\left(\boldsymbol{\Gamma}_{1, j}, t\right) \mathrm{d} \gamma_{1, j} \\
P_{B}(\mathrm{t})= & \int_{\gamma_{1,1}^{*}}^{1} p_{1}\left(\boldsymbol{\Gamma}_{1, j}, t\right) \mathrm{d} \gamma_{1, j} \\
& +\sum_{j=1}^{2} \int_{0}^{\gamma_{2, j}^{*}} p_{2}\left(\boldsymbol{\Gamma}_{2, j}, t\right) \mathrm{d} \gamma_{2, j} \\
P_{C}(\mathrm{t})= & \int_{\gamma_{2,1}^{*}}^{1} p_{1}\left(\boldsymbol{\Gamma}_{1,1}, t\right) \mathrm{d} \gamma_{1, j} \\
& +\sum_{j=1}^{2} \int_{0}^{\gamma_{3, j}^{*}} p_{3}\left(\boldsymbol{\Gamma}_{3, j}, t\right) \mathrm{d} \gamma_{3, j} \\
P_{D}(\mathrm{t})= & \int_{\gamma_{2,1}^{*}}^{1} p_{2}\left(\boldsymbol{\Gamma}_{2,1}, t\right) \mathrm{d} \gamma_{2,1} \\
P_{E}(\mathrm{t})= & \int_{\gamma_{2,2}^{*}}^{1} p_{2}\left(\boldsymbol{\Gamma}_{2,2}, t\right) \mathrm{d} \gamma_{2,2} \\
P_{F}(\mathrm{t})= & \int_{\gamma_{3,1}^{*}}^{1} p_{3}\left(\boldsymbol{\Gamma}_{3,1}, t\right) \mathrm{d} \gamma_{3,1} \\
P_{G}(\mathrm{t})= & \int_{\gamma_{3,2}^{*}}^{1} p_{3}\left(\boldsymbol{\Gamma}_{3,2}, t\right) \mathrm{d} \gamma_{3,2}
\end{aligned}
$$

donde $\gamma_{i, j}^{*}$ representan los valores de $\gamma$ que maximizan la el potencial $\phi_{i}$, o en otras palabras el estado de transición.

\section{RESULTADOS Y DISCUSIONES}

De acuerdo a las simulaciones realizadas, se obtuvieron las probabilidades asociadas a cada estructura en el sistema. La Figura 2 muestra las barreras potenciales en cada espacio para cada trayectoria posible. En este caso, para valores de $\gamma_{i, j}$ que no pertenezcan a una trayectoria, el potencial (o barrera potencial) es infinito.

La Figura 3 muestra las probabilidades globales de encontrar cada una de las estructuras. A partir de los resultados se verifica la conservación del número de bloques en el sistema (la probabilidad se conserva), i.e, la suma de las probabilidades globales de los componentes es igual a uno para todo tiempo. En la Figura 3 se muestra la dinámica del ensamble de trímeros mediante el modelo propuesto y se observa que los trímeros más probables corresponden a los que están más favorecidos termodinámicamente (menor potencial químico estándar).
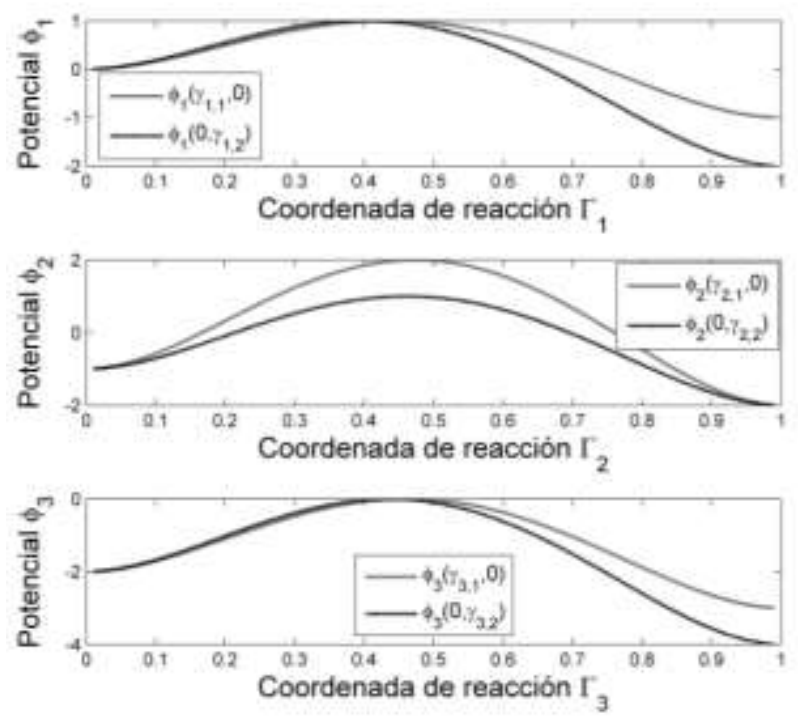

Figura 2. Barreras de energía potencial, en función de la coordenada de reacción en cada espacio, usadas para la solución numérica del sistema de ecuaciones. Fuente. Elaboración propia.

Adicionalmente, se observa que para tiempos iniciales es muy probable encontrar el dímero $B$, ya que los trímeros formados a partir de este no son tan favorecidos termodinámicamente y por tanto es poco probable el consumo del dímero $B$. Por otra parte, es poco probable encontrar el dímero $C$, ya que los trímeros que se forman a partir de este dímero son bastante probables, por tanto se consume en gran cantidad el dímero $C$.

\section{CONCLUSIONES}

A partir de los potenciales energéticos (Figura 2) es posible resolver el sistema de ecuaciones (Ecuación 1) para obtener la probabilidad de encontrar cada estructura en el sistema (Figura 3). Así, a partir del sistema de ecuaciones tipo Fokker-Planck propuesto, acoplado por sus condiciones de frontera, se describió la dinámica del ensamble de trímeros no-simétricos en los espacios $\boldsymbol{\Gamma}_{i}$. 
Esta propuesta para resolver ensambles de estructuras se constituye en una valiosa herramienta para desarrollar métodos de simulación, dirigidos a estudiar sistemas fisicoquímicos fuera del equilibrio en donde se ensamblan estructuras. Finalmente, esta propuesta puede presentar ventajas relacionadas con el tiempo de cómputo frente a métodos basados en Dinámica Molecular y Montecarlo, por el hecho de no requerir de un gran número de partículas para la descripción de sistemas en la mesoescala.

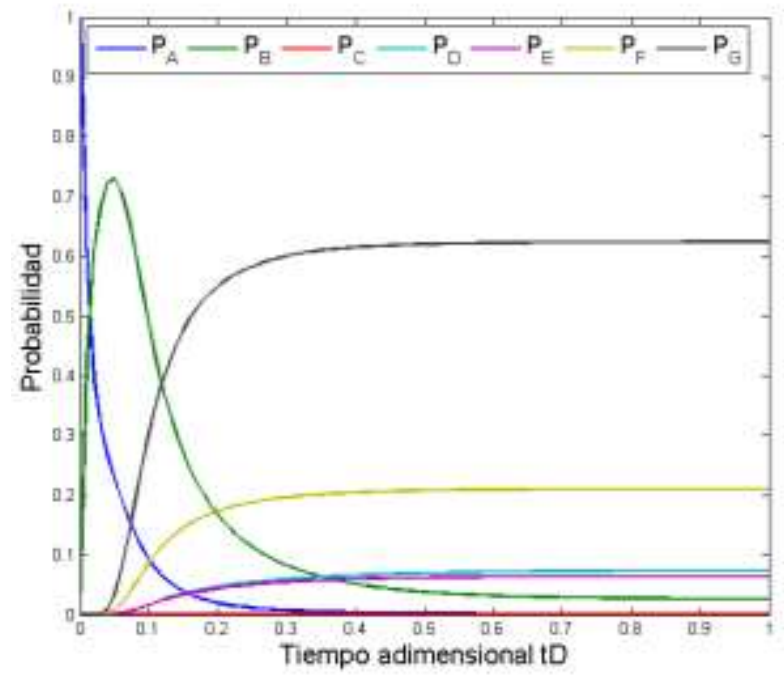

Figura 3. Probabilidades globales de encontrar cada estructura en función del tiempo adimensional. Fuente. Elaboración propia.

A partir de la presente propuesta también se podría estudiar el desensamble de moléculas sencillas y sus posibles trayectorias de desensamble. Por otra parte, el estudio y análisis de la termodinámica y la cinética del ensamble y desensamble (o descomposición) de polímeros lineales [11] y algunos polímeros sencillos de importancia económica, como el ácido poliláctico [13], biomasa lignocelulosicas [14] y las parafinas [15], podrían llevarse a cabo para estudiar estas estructuras fuera del equilibrio termodinámico. Sin embargo, aunque en el caso de polímeros lineales el presente método ha otorgado muy buenos resultados [11], deben de construirse los potenciales energéticos para cada tipo de polímero en medios no ideales.

\section{AGRADECIMENTOS}

Este trabajo fue parcialmente financiado por parte de la Facultad de Ciencias de la Universidad Nacional de Colombia sede Medellín.

\section{REFERENCIAS}

[1] B. Grzybowski, C. Wilmer, J. Kim, K. Browne and K. Bishop, "Self-assembly: from crystals to cells," Soft Matter, vol. 5, no. 6, pp. 1110, Feb., 2009.

[2] M. Fialkowski, K. Bishop, R. Klajn, S. Smoukov, C. Campbell and B. Grzybowski, "Principles and Implementations of Dissipative (Dynamic) SelfAssembly," The Journal of Physical Chemistry B, vol. 110, no. 6, pp. 2482-2496, Mar., 2006.

[3] J. Lehn, "Toward Self-Organization and Complex Matter," Science, vol. 295, pp.2400-2403, Mar., 2002.

[4] G. M. Whitesides and B. A. Grzybowski, "SelfAssembly at All Scales," Science, vol. 295, pp.24182421,Mar., 2002.

[5] R. Greenberg and A. Brahic, Planetary rings. Tucson, Arizona: The university of Arizona Press, 1984.

[6] S. Mann, "Self-assembly and transformation of hybrid nano-objects and nanostructures under equilibrium and nonequilibrium conditions," Nature Materials, vol. 8, no. 10, pp. 781-792, Oct., 2009.

[7] K. Tretiakov, K. Bishop and B. Grzybowski, "The dependence between forces and dissipation rates mediating dynamic self-assembly," Soft Matter, vol. 5, no. 6, p. 1279, Ene., 2009.

[8] K. Tretiakov, I. Szleifer and B. Grzybowski, "The Rate of Energy Dissipation Determines Probabilities of Non-equilibrium Assemblies," Angewandte Chemie, vol. 125, no. 39, pp. 10494-10498, Sep., 2013.

[9] P. Jha, V. Kuzovkov,B. Grzybowski andM. Olvera de la Cruz, "Dynamic self-assembly of photo-switchable nanoparticles," Soft Matter, vol. 8, no. 1, pp. 227-234, Nov., 2011.

[10] D. Reguera, J. Rub'1 and J. Vilar, "The Mesoscopic Dynamics of Thermodynamic Systems," The Journal of Physical Chemistry B, vol. 109, no. 46, pp. 21502-21515, Oct., 2005.

[11] A. Arango-Restrepo, "Non-equilibrium selfassembly processes: Thermodynamics of structures formation out of equilibrium," Tesis de Máster, Dept. Química, Universidad Nacional de Colombia, Medellín, Colombia, 2017. 
[12] H.A. Kramers, "Brownian Motion in a Field of Force and the Diffusion Model of Chemical Reactions," Physica, vol. 7, no. 4, pp. 284-304, Abr., 1940.

[13] H. Estupíñan, C. Vásquez, L.F. Ardila, "Degradación de ácido poliláctico / hidroxiapatita y ácido poliglicólico en fluido corporal simulado," Rev. UIS Ing., vol. 10, no. 2, pp. 145-150, 2011.

[14] Y. Rueda-Ordoñez, K. Tannous, "Análisis cinético de la descomposición térmica de Biomasa Aplicando un Esquema de Reacciones Paralelas Independientes," Rev. UIS Ing., vol. 16, no. 2, pp. 119-127, 2017.

[15] D. P. González, C. A. Villabona, H. R. Vargas, E. Ariza, C. E. Roa, C. Brajas, "Métodos para el Control e Inhibición de la Acumulación de Depósitos Parafínicos," Rev. UIS Ing., vol. 9, no. 2, pp. 193-206, 2010. 